\title{
Fishing for an antidote to cyanide
}

The use of animals as sentinels for exposure to toxins is not new but has come a long way since the first canary was brought into a coal mine. A recent twist took the sentinel from airborne to aquatic, establishing three key results: first, zebrafish are a viable model for cyanide exposure in humans. Second, zebrafish provide an excellent medium for high-throughput chemical screening. And third, riboflavin can mitigate the harmful effects of cyanide toxicity in zebrafish. The research was done by Anjali Nath and Randall Peterson (Harvard Medical School, Charlestown, MA, and Broad Institute, Cambridge, MA) with colleagues at the University of California (Irvine and San Diego) and the US Army Medical Research Institute of Chemical Defense (Aberdeen, MD). Future work will test whether riboflavin also acts as a cyanide antidote in mammalian models. In a press release, Peterson said, "Hopefully, the cyanide biomarkers and antidotes we discover with the help of zebrafish can one day improve our ability to diagnose and treat humans affected by cyanide poisoning."

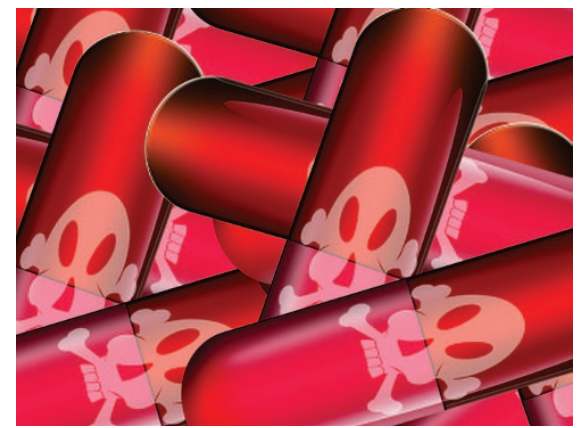

concentrations in all three species, suggesting that inosine level could be a valid biomarker for cyanide exposure, potentially useful in both diagnosis and antidote development.

Next, the researchers developed a highthroughput, in vivo, whole-organism assay to systematically test whether thousands of compounds could protect zebrafish against cyanide toxicity (FASEB J. 27, 1928-1938; 2013). They screened 3,120 candidate drugs and identified four that rescued zebrafish from otherwise lethal doses of cyanide: cisplatin, carboplatin, methotrexate and riboflavin. The first three compounds are chemotherapeutic agents, whereas riboflavin is a vitamin. Riboflavin was the most effective antidote of the four and did not appear to have negative side effects in fish or humans, unlike the other three compounds.

In further tests, riboflavin mitigated the effects of cyanide on neurobehavioral and metabolic function in the fish. "Riboflavin normalizes many of the cyanide-induced neurological and metabolic perturbations in zebrafish," the article states.

Monica Harrington

\section{MEMORIES OBSERVED IN ZEBRAFISH BRAINS}

Isolating the neural circuits responsible for long-term memory formation, storage and retrieval requires an experimental system to observe patterns of neural activity across the whole brain during behavior. Zebrafish, which have transparent brains, provide such a system.

A team of researchers at RIKEN Brain Science Institute (Wako, Japan) used transgenic zebrafish that expressed a calcium-sensitive protein genetically engineered to glow less brightly when calcium levels increase, which occurs when neurons fire. Studying the activity of these proteins under a microscope allowed them to measure neural activity across the entire brain in real time.

The researchers, led by Hitoshi Okamoto, trained the fish to perform an avoidance task. The fish were placed in a shuttle tank divided into two compartments by a hurdle through which they could swim to the other compartment. A red light was presented in the compartment where the fish was located. If the fish did not go to the opposite compartment before the light turned off, it would be given a punishment of a mild electric shock.

Thirty minutes after the training, the team observed the brains of the zebrafish while a red light was switched on and off, and they only saw activity in the optic tectum, a brain region responsible for processing vision. But 24 hours later, a pattern of activity was observed in the telencephalon, an area believed to correspond to the mammalian neocortex. To test whether the telencephalon was necessary for remembering how to perform the avoidance task, the researchers impaired this brain area before and after the training in a new group of fish. The impaired fish could learn the avoidance task, and they could remember how to perform the task 30 minutes later. But 24 hours later, the fish had forgotten what they had learned, confirming that the neurons in the telencephalon were necessary for long-term memory (Neuron doi:10.1016/j.neuron.2013.04.009; published online 16 May 2013).

In follow-up experiments, fish that had previously learned to swim away from the red light were retrained to stay put in their compartment to avoid the shock. When they learned this new behavior, the scientists observed a brand new pattern of activity in the telencephalon. Yet 24 hours later, the retrained fish reverted to the avoidance task they had originally learned, and their pattern of activity reverted as well. "That may tell us something very important about how the memory mechanism works," says 0 kamoto. 